\section{Warum die EU an einer europäischen Cloud tüftelt}

\author{
Die Coco Cloud der Europäischen Kommission soll Kunden \\ maximale Vertraulichkeit und Sicherheit garantieren.
}

Die Europäische Kommission möchte die in der Cloud gespeicherten Daten europäischer Kunden besser schützen. Um das zu erreichen, müssen die Daten erst einmal in den Binnenraum der EU zurückgeholt werden, um sie mit verbindlichen Sicherheitsstandards geschützt zu speichern. In Europa soll daher eine neue Infrastruktur für sicheres und gesetzeskonformes Cloud Computing entstehen: die Coco Cloud. Sie steht für Confidential Compliant Cloud. Rechtliche Unterschiede und Regulierungsprobleme zwischen EU-Ländern will das Projekt überbrücken und einen einheitlichen Sicherheitsstandard etablieren. Gleichzeitig ist es das Ziel des Projekts, die Cloud so flexibel zu gestalten, dass Unternehmen und Endanwender wie gewohnt von überall und von jedem Gerät auf ihre Daten zugreifen und weitere Anwendungen und Services einbinden können. Mehr dazu auf:

Sif www.springerprofessional.de/5155256

\section{Sicherheit ist die größte Hürde für eine Mobile-Strategie}

\author{
Immer mehr Mitarbeiter nutzen eigene mobile Devices für \\ berufliche Tätigkeiten. Doch das Sicherheitsrisiko ist hoch.
}

Mobile Geräte unterstützen den Außendienst bei der Präsentation und im Verkauf. Sie sorgen dafür, dass Mitarbeiter auf Geschäftsreisen erreichbar sind und auf Daten im Netzwerk zugreifen können. Lassen Unternehmen auch private Smartphones und Tablets der Mitarbeiter zu („Bring Your Own Device“, BYOD), sinken die IT-Kosten. Unternehmen erschließen obendrein neue Verkaufskanäle via Mobile Commerce und gestalten die Prozesse in der Produktion mit mobilen Geräten. Dies birgt aber auch Risiken. Eine Hürde sind vor allem Sicherheitsbedenken, angefangen beim Datenschutz bis zur Kontrolle der Netzwerkzugriffe. Erst 56 Prozent der großen und mittelständischen Unternehmen haben bisher eine umfassende Strategie für ihr mobiles Geschäft entwickelt, so die Trendstudie „Mobile Enterprise Review“ von Lünendonk. Für BYOD fehlen jedoch häufig die rechtlichen Grundlagen. Unternehmen tun deshalb gut daran, in Abstimmung mit dem Betriebsrat klare Regeln für den Umgang mit privaten und geschäftlichen Daten zu entwickeln. Mehr dazu auf:

Sf www.springerprofessional.de/5130278

\section{Springer Gabler}

\section{Entrepreneurial Business Modeling}

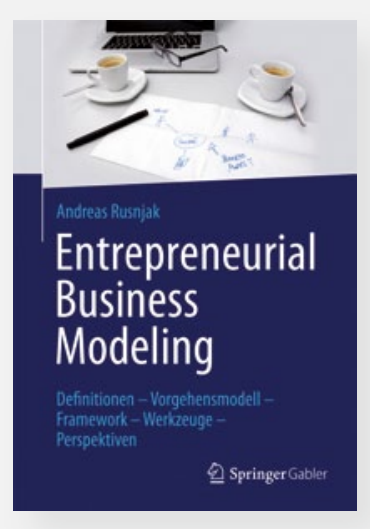

Andreas Rusnjak

Entrepreneurial Business Modeling

Definitionen - Vorgehensmodell Framework - Werkzeuge Perspektiven

- Fundierte Betrachtung des Themas Business Modeling im Kontext einer erfolgsfaktorenorientierten Strategieentwicklung

- Aktuelle Erkenntnisse aus der Geschäftsmodellentwicklung

- Umfassende Darstellung mit zahlreichen Fallbeispielen

2014. IV, 241 S. 109 Abb. Geb. $€$ (D) $44,99|€(A) 46,25|^{*} \mathrm{sFr} 56,00$ ISBN 978-3-658-03766-6

$€(D)$ sind gebundene Ladenpreise in Deutschland und enthalten $7 \%$ MwSt. $€(A)$ sind gebundene Ladenpreise in Österreich und enthalten $10 \%$ MwSt Die mit * gekennzeichneten Preise sind unverbindliche Preisempfehlungen und enthalten die landesübliche MwSt. Preisänderungen und Irrtümer vorbehalten.

springer-gabler.de 